\title{
Validation of the Estimation of Physiologic Ability and Surgical Stress (E-PASS) Score in Liver Surgery
}

\author{
Vanessa M. Banz • Peter Studer · Daniel Inderbitzin · \\ Daniel Candinas
}

Published online: 17 March 2009

(C) Société Internationale de Chirurgie 2009

\begin{abstract}
Background The estimation of physiologic ability and surgical stress (E-PASS) has been used to produce a numerical estimate of expected mortality and morbidity after elective gastrointestinal surgery. The aim of this study was to validate E-PASS in a selected cohort of patients requiring liver resections (LR).

Methods In this retrospective study, E-PASS predictor equations for morbidity and mortality were applied to the prospective data from 243 patients requiring LR. The observed rates were compared with predicted rates using Fisher's exact test. The discriminative capability of E-PASS was evaluated using receiver-operating characteristic (ROC) curve analysis.

Results The observed and predicted overall mortality rates were both $3.3 \%$ and the morbidity rates were 31.3 and $26.9 \%$, respectively. There was a significant difference in the comprehensive risk scores for deceased and surviving patients $(p=0.043)$. However, the scores for patients with or without complications were not significantly different $(p=0.120)$. Subsequent ROC curve analysis revealed a poor predictive accuracy for morbidity.

Conclusions The E-PASS score seems to effectively predict mortality in this specific group of patients but is a poor predictor of complications. A new modified logistic regression might be required for LR in order to better predict the postoperative outcome.
\end{abstract}

V. M. Banz · P. Studer · D. Inderbitzin - D. Candinas ( $\square)$ Department of Visceral Surgery and Medicine, Inselspital, Bern University Hospital and University of Bern, CH-3010 Bern, Switzerland

e-mail: daniel.candinas@insel.ch

V. M. Banz

e-mail: vanessa.banz@gmx.ch

\section{Introduction}

The quality of medical care is being increasingly judged by hard facts and parameters that can be measured and compared, including the length of hospital stay, in-hospital mortality and morbidity, and the costs generated [1, 2]. If potential postoperative problems can be predicted in patient subgroups based on pre- and intraoperative measures, adequate preemptive steps can be taken to avoid these complications. Mortality and morbidity are, if well defined, readily measurable and objective parameters for monitoring the standard of care within a center while equally allowing for comparisons between different centers.

The estimation of physiologic ability and surgical stress (E-PASS) score was initially developed to predict adverse postoperative effects in a study population of approximately 300 patients requiring elective gastrointestinal surgery, ranging from laparoscopic cholecystectomy to transthoracic esophagectomy [3]. Based on their E-PASS score, patients are categorized into five groups, which then allows for risk stratification of the expected morbidity and mortality. The E-PASS score has already been validated and shown to be reproducible by other authors, not only for gastrointestinal surgery, but also for the elective repair of abdominal aortic aneurysms, thoracic surgery, and osteosynthesis for hip fractures [4-7]. A possible advantage of the E-PASS scoring system includes better overall assessment that not only permits the evaluation of a patient's preoperative reserve capacities, but also allows for a concise judgment of the surgical stress applied. Ideally, the surgeon can make a rough preoperative estimate of how much "surgical stress" the patient can tolerate in order to obtain a low E-PASS score, which is associated with a low expected morbidity and mortality. 
The aim of the current study was to review whether the E-PASS scoring system could be used, without restrictions, in hepatic surgery as a means of correctly predicting morbidity and mortality.

\section{Materials and methods}

We carried out a retrospective analysis of prospective data collected between January 2002 and December 2006 and entered into a computer database system. All patients requiring hepatic resection for benign or malignant conditions treated in our unit were included for analysis. Exclusion criteria included patients who were initially seen for major hepatic surgery but only received liver biopsies due to disseminated, inoperable disease. All patients requiring emergency hepatic surgery were also excluded from further analysis; they may already have met, to an extent, the criteria for systemic inflammatory response syndrome (SIRS) [8], possibly confounding the E-PASS scores. The exclusion of patients requiring emergency surgery was in accordance with the initial guidelines developed by Haga et al. [3]. During the defined study period, a total of 243 patients were included for E-PASS analysis.

Postoperative complications were defined as all problems requiring medical, surgical, or other intervention and treatment [9-11]. Documented general and liver-specific complications included superficial infections in the form of erythema or discharge requiring opening of the wound or antibiotic therapy; deep infections in the form of intraabdominal collections or an abscess confirmed radiologically or at laparotomy; pulmonary embolus or thrombosis confirmed radiologically by computed tomography or duplex sonography; pneumonia determined by typical clinical presentation, auscultatory findings, or positive chest X-ray; delayed gastric emptying requiring the placement of a nasogastric tube and intravenous fluids for more than 1 week with delayed patient discharge and hemorrhage or hematoma seen as a distinct drop in hemoglobin values confirmed clinically, radiologically, or at laparotomy; biliomas in the form of intra-abdominal fluid collection with clearly elevated bilirubin values requiring percutaneous drainage or surgical intervention.

Classification using the criteria of the American Society of Anesthesiologists (ASA) was carried out by the attending anesthetist in charge prior to surgery. The operation time was defined as the time from the first skin incision to complete closure of all wounds. The E-PASS scoring system was used retrospectively with the computer database and supplemented by the patients' medical files if necessary, according to the defined criteria [3]. The comprehensive risk score (CRS) was calculated using the E-PASS equations and includes the calculation of the preoperative risk score (PRS) and the surgical stress score (SSS) (Table 1). Patients were divided into one of five groups [12] according to their final CRS: Group 1, CRS $<0$; Group 2, 0 to $<0.5$; Group 3, 0.5 to $<1.0$; Group 4,

Table 1 Equations for calculating the E-PASS score [12]

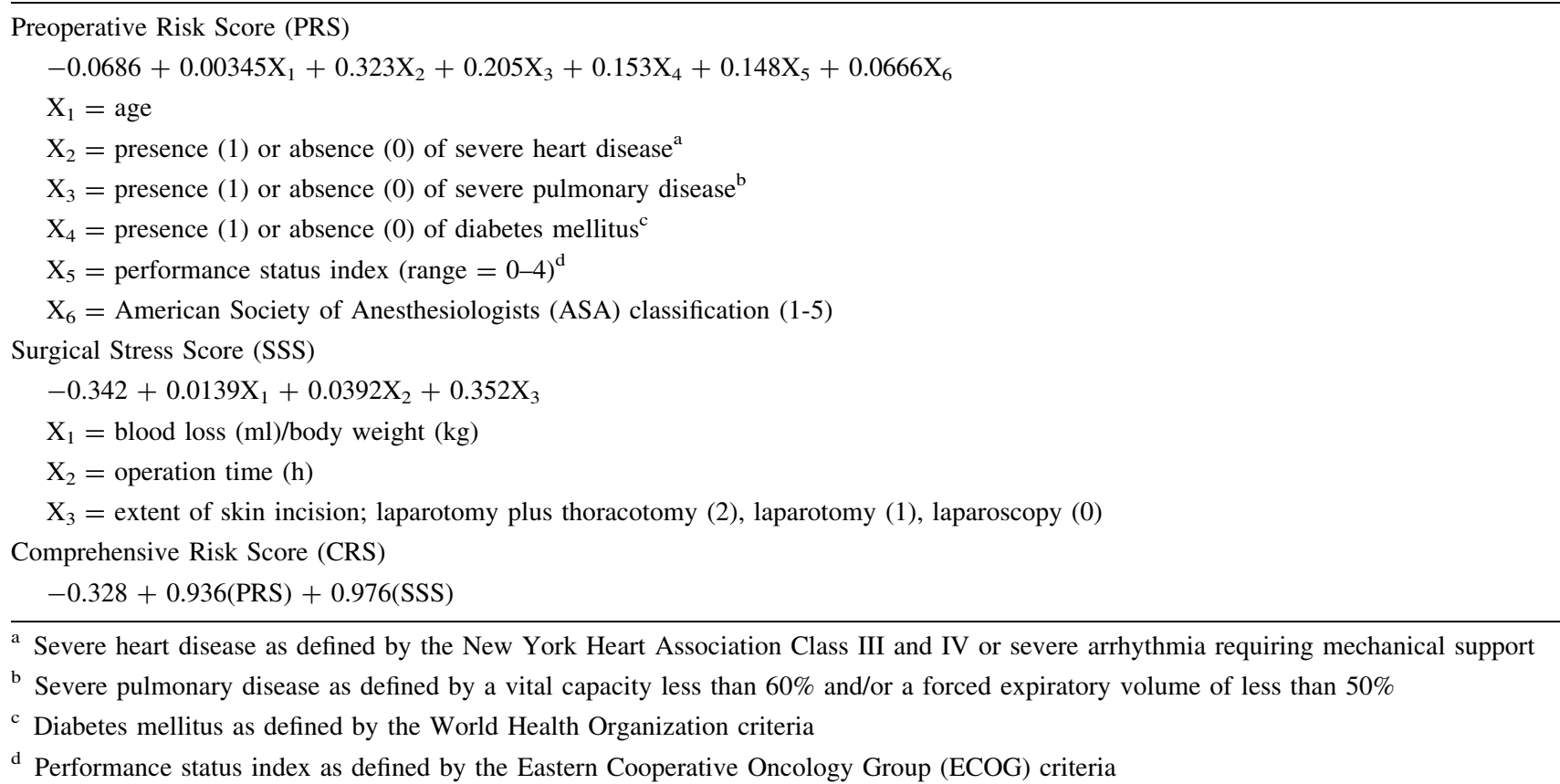


1.0 to $<1.5$; and Group 5, $\geq 1.5$. Patients in Group 1 all have a CRS value below zero, i.e., only negative CRS numbers are included. Patients in Group 1 have the lowest risk of postoperative complications or death, whereas patients in Group 5 have the highest risk. Hospital mortality was defined as death during the same admission as the operation.

To avoid possible errors of observer-specific mistakes, all data were independently checked and compared by two staff members of our unit.

\section{Statistical analysis}

All variables were analyzed descriptively by the Fisher's exact test or Wilcoxon rank sum test; the Mann-Whitney $\mathrm{U}$ test was used to examine differences in CRS distribution of patients with and without complications. The same test was applied for the mortality group. Multivariate analysis was performed using the multiple logistic regression model. The ROC curves were plotted to assess the extent to which CRS, PRS, and SSS can accurately predict complications, where morbidity and mortality are combined, and the area under the curve (AUC) was used as a measure of overall diagnostic accuracy. A two-sided exact binomial test was used to compare CRS groupspecific effective morbidity with expected morbidity. A $p$ value of less than 0.05 was taken to be significant. Statistical calculations were carried out using $\mathrm{R}$ version 2.5 and SAS version 9.1 software. All statistical analyses were done with professional help from the Institute of Mathematical Statistics and Actuarial Science, University of Bern, Bern, Switzerland.

\section{Results}

Six of the initial 249 patients $(2.4 \%)$ had to be excluded from analysis because they either had incomplete data or met SIRS criteria prior to surgery This resulted in a total of 243 patients receiving hepatic resections in our department who were analyzed. Patient demographics and admission data are summarized in Table 2. Table 3 lists the operative procedures performed. One hundred eighty-eight $(77.4 \%)$ patients were operated on for malignant disease, with one third requiring hepatic resection for colorectal liver metastases (Table 4). Only a small percentage of our patients underwent surgery for hepatocellular carcinoma, with 21 patients $(8.6 \%)$ of the total study population suffering from cirrhosis.

Both the observed and predicted overall mortality rates were $3.3 \%$ ( 8 and 8.03 patients, respectively); the observed and predicted overall morbidity rates were $31.3 \%$ (76 patients) and $26.9 \%$ (65.4 patients), respectively. Overall
Table 2 Demographics of the 243 patients included in the E-PASS study

\begin{tabular}{|c|c|}
\hline Variable & $N(243)$ \\
\hline Median age (range) (years) & $61(19-82)$ \\
\hline Sex ratio $(M: F)$ & $131: 112$ \\
\hline ASA classification (I:II:III) & $14: 115: 114$ \\
\hline Mean (range) BMI $\left(\mathrm{kg} / \mathrm{m}^{2}\right)$ & $24(16.8-40.6)$ \\
\hline Severe heart disease $(\%)^{\mathrm{a}}$ & $32(13.2)$ \\
\hline Severe pulmonary disease $(\%)^{\mathrm{b}}$ & $31(12.8)$ \\
\hline Diabetes mellitus $(\%)^{\mathrm{c}}$ & $29(11.9)$ \\
\hline Observed hospital morbidity (\%) & $76(31.3)$ \\
\hline Observed hospital mortality (\%) & $8(3.3)$ \\
\hline Observed 30-day mortality (\%) & $6(2.5)$ \\
\hline
\end{tabular}

ASA American Society of Anesthesiologists; BMI body mass index

a Severe heart disease as defined by the New York Heart Association Class III and IV or severe arrhythmia requiring mechanical support

b Severe pulmonary disease as defined by a vital capacity less than $60 \%$

c Diabetes mellitus as defined by the World Health Organisation criteria

Table 3 Extent of liver resection in 243 patients

\begin{tabular}{lc}
\hline Type of hepatic resection & $N(\%)$ \\
\hline Hemihepatectomy left & $17(7)$ \\
Extended hemihepatectomy left & $13(5.4)$ \\
Hemihepatectomy right & $62(25.5)$ \\
Extended hemihepatectomy right $^{\text {Atypical segment }}{ }^{\mathrm{a}}$ resection $(\geq 1)$ & $18(7.4)$ \\
Typical segment $^{\mathrm{a}}$ resection $(\geq 1)$ & $23(9.5)$ \\
Laparoscopic resection $^{\mathrm{a}}$ & $106(43.6)$ \\
$\geq 2$ segments & $4(1.6)$ \\
$<2$ segments $^{\mathrm{a}}$ & $185(76)$ \\
\hline
\end{tabular}

${ }^{a}$ Liver segments are based on Couinaud's classification

group-specific (Groups 1-5) predictive correlation was good with a correlation coefficient of 0.96. Complications were observed in 76 patients (Table 5) of which 10 (13.2\%) required operative intervention, including four abdominal lavages for biliomas and one for an abscess, one revision for a biliary-cutaneous fistula and one for a hematoma, one redo hepaticojejunostomy for persistent biliary leakage, one closure of a small duodenal perforation, and one ileostomy for a colonic anastomotic leakage in a patient who had received a combined liver and colon segment resection. Of the eight deceased patients, five died of multiple-organ failure, one of postoperative liver failure, and two of acute cardiac failure.

Table 6 summarizes the mean CRS, PRS, and SSS values for the study population, specifically comparing those with and without mortality or morbidity. Expected 
Table 4 Indications for hepatic resection

\begin{tabular}{lll}
\hline Underlying liver pathologies & $N=243(\%)$ & Patients with cirrhosis (Child score) \\
\hline Colorectal liver metastases & $78(32.1)$ & 1 (Child A) \\
Other liver metastases & $40(16.5)$ & 0 \\
Benign liver tumors & $38(15.6)$ & 0 \\
Cholangiocarcinoma & $34(14.0)$ & 1 (Child A) \\
Hepatocellular carcinoma & $24(9.9)$ & $19(13$ Child A, 6 Child B) \\
Echinococcal cysts & $17(7.0)$ & 0 \\
Other primary malignant tumors & $12(4.9)$ & 0 \\
\hline
\end{tabular}

Table 5 Postoperative complications requiring medical, surgical, or other intervention

\begin{tabular}{lc}
\hline Type of complication & $N(\%)^{\mathrm{a}}$ \\
\hline Bilioma/ascites & $36(14.8)$ \\
Superficial infection & $16(6.6)$ \\
Pneumonia & $11(4.5)$ \\
Deep infection & $10(4.1)$ \\
Pulmonary embolus & $8(3.3)$ \\
Hemorrhage & $7(2.9)$ \\
Delayed gastric emptying & $5(2.1)$ \\
Deep vein thrombosis & $2(0.8)$ \\
Other (including myocardial infarction, percutaneous bile & $6(2.5)$ \\
$\quad$ fistula, duodenal perforation, leakage of colon & \\
anastomosis, urosepsis, and portal vein thrombosis) & \\
Number of complications & 101 \\
Number of patients with complications & $76(31.3)$ \\
Complications requiring operative intervention & $10(13.2)$
\end{tabular}

${ }^{\mathrm{a}}$ Out of a total 243 patients

morbidity (or mortality) was calculated by examining each of the five possible CRS groups individually and multiplying the number of patients in each group (as observed in this patient population) by the percentage probability of a complication (or death) occurring according to the values given by Haga's study population [12].

As might be expected, deceased patients had a significantly higher CRS than surviving patients $(p=0.043)$. The CRS revealed no significant difference between the rounded expected and observed in-hospital mortality ( $p=0.804)$, indicating that it might effectively predict the outcome. However, there was no significant difference in the CRS of patients with or without morbidity $(p=0.120)$. The lack of power of CRS for predicting postoperative complications is also demonstrated by the low AUC (0.574) (Fig. 1a). Similar results were obtained by a separate analysis of PRS and SSS (Fig. 1b and c, $\mathrm{AUC}=0.521$ and 0.571 , respectively).

When comparing the group distribution of our 243 patients to that of the 5215 patients in Haga's study population [12], our study population exhibits a highly significant right shift $(p<0.001)$, with more patients belonging to a higher CRS group (Fig. 2). If group-specific
Table 6 Summary of preoperative risk score (PRS), surgical stress score (SSS), and comprehensive risk score (CRS) for liver resection patients

\begin{tabular}{|c|c|c|c|c|}
\hline Score & Group & $N$ & Mean (SD) & $p$ value* \\
\hline \multirow[t]{7}{*}{ PRS } & All patients & 243 & $0.44(0.20)$ & \\
\hline & Mortality & & & \\
\hline & Yes & 8 & $0.54(0.21)$ & 0.150 \\
\hline & No & 235 & $0.44(0.20)$ & \\
\hline & Morbidity & & & \\
\hline & Yes & 76 & $0.44(0.21)$ & 0.866 \\
\hline & No & 158 & $0.44(0.20)$ & \\
\hline \multirow[t]{7}{*}{ SSS } & All patients & 243 & $0.37(0.29)$ & \\
\hline & Mortality & & & \\
\hline & Yes & 8 & $0.50(0.38)$ & 0.477 \\
\hline & No & 235 & $0.36(0.29)$ & \\
\hline & Morbidity & & & \\
\hline & Yes & 76 & $0.42(0.36)$ & 0.090 \\
\hline & No & 158 & $0.34(0.24)$ & \\
\hline \multirow[t]{7}{*}{ CRS } & All patients & 243 & $0.44(0.34)$ & \\
\hline & Mortality & & & \\
\hline & Yes & 8 & $0.67(0.45)$ & 0.043 \\
\hline & No & 235 & $0.44(0.34)$ & \\
\hline & Morbidity & & & \\
\hline & Yes & 76 & $0.50(0.38)$ & 0.120 \\
\hline & No & 158 & $0.41(0.31)$ & \\
\hline
\end{tabular}

$* p$ values were calculated using the Wilcoxon signed-rank test. Deceased patients were excluded from morbidity analysis. $p<0.05$ was considered significant

morbidity is evaluated, comparing actual, i.e., observed, morbidity with expected morbidity (Table 7), we found that significantly more patients in Group 2 (CRS 0 to $<0.5)$ have complications than would be expected $(p=0.001)$. No other group had significant differences in observed and expected morbidity.

To determine whether the PRS or SSS plays a more important predictive role, we performed a multiple logistic regression analysis with postoperative complications as the dependent variable and PRS, SSS, and their interaction as independent variables. Since the interaction term was not significantly different from zero $(p=0.321)$, we estimated 

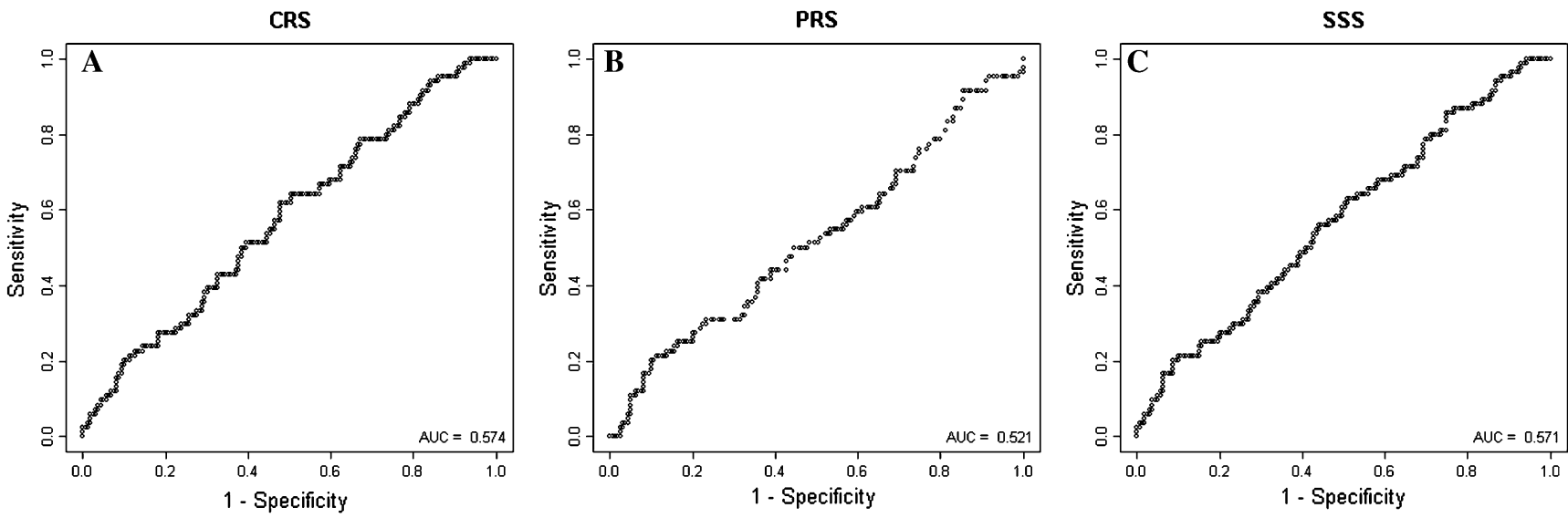

Fig. 1 Empirical receiver operating characteristic (ROC) curves to assess the extent to which the comprehensive risk score (CRS), preoperative risk score (PRS), and surgical stress score (SSS) can accurately predict postoperative complications. The area under the curve (AUC) is used as a measure of overall diagnostic accuracy. a
CRS does not accurately predict morbidity with a low AUC of 0.574 . b The AUC is very low (0.521), indicating that the PRS is poor in predicting morbidity. $\mathbf{c}$ The AUC is 0.571 , a low value, indicating the poor predictive power of the SSS as far as morbidity is concerned

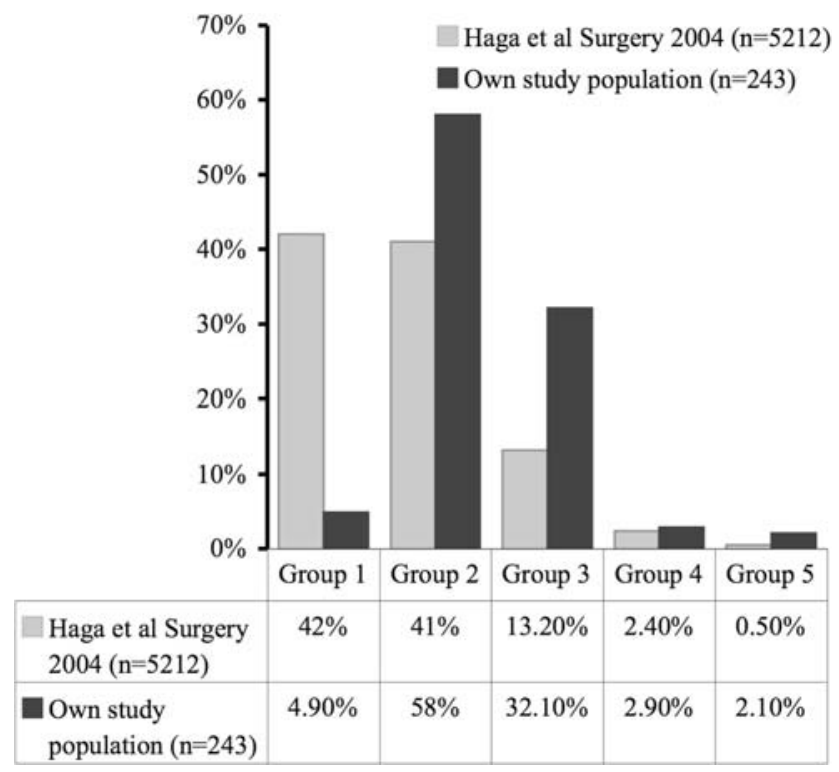

Fig. 2 The 243 patients from the current study compared to the study population of 5212 patients from Haga et al. [12]. Our study population shows a right-shift with significantly more patients in higher CRS groups $(p<0.001)$, indicating that our population consisted of more high-risk patients. Group 1, CRS $<0.0$; Group 2, CRS 0.0 to $<0.5$; Group 3, 0.5 to $<1.0$; Group 4, 1.0 to $<1.5$; Group 5, $>1.5$. Note that $<0.0$ includes all CRS values below zero, i.e., negative numbers only

a logistic regression model without interaction. In this model, the PRS was not significantly different from zero $(p=0.578)$; however, the SSS was a significant variable $(p=0.022)$. In a third step, we used a model with SSS as the only independent variable and it was still significant $(p=0.021)$. Therefore, in the context of liver surgery, the SSS plays a more important predictive role than the PRS.
Comparing the PRS and SSS of patients with or without complications revealed no overall significant difference ( $p=0.866$ and 0.090 , respectively), but group-specific comparisons showed a lack of fit for Groups 1, 2, and 4 .

\section{Discussion}

This is the first time the E-PASS scoring system has been applied to a specific hepatobiliary surgical patient population. In our institution, the E-PASS system fails to correctly predict patient outcome with respect to morbidity, as demonstrated by the low AUC. If one breaks down the E-PASS scoring system into the two separate contributors, namely, the PRS and the SSS, the predictive strength of either one is poor.

If mortality is looked at by itself, the CRS does seem to correlate with the risk of death $(p=0.043)$. However, these results need to be interpreted with caution because the mortality rate corresponds to only eight patients, resulting in a very small group for analysis. However, we can show that the SSS seems to bear more weight than the PRS. This would imply that surgical stress needs to be kept to a minimum rather than exempting patients from further surgery based solely on their preoperative status or PRS. The authors of an initial E-PASS study also argued that the SSS is potentially better correlated with postoperative complications than the PRS, although this tended to be the case only in younger patients [3].

With a postoperative morbidity rate of $31.3 \%$ and an inhospital mortality rate of $3.3 \%$, our institution lies within the accepted range of complications after hepatic resections [13-15]. However, the risk of postoperative morbidity was 
Table 7 Group-specific comparisons of expected and observed morbidity by comprehensive risk score (CRS)

\begin{tabular}{lcccrr}
\hline CRS group & Number of patients & Expected morbidity $\left.^{\text {a }} \%\right)$ & Expected morbidity $(n)^{\text {Effective morbidity }(n)}$ & $p^{*}$ \\
\hline $1(<0)$ & 12 & 4.5 & 1 & 1 & 0.425 \\
$2(0$ to $<0.5)$ & 138 & 20.4 & 28 & 44 & 0.001 \\
$3(0.5$ to $<1.0)$ & 75 & 40.2 & 30 & 27 & 0.482 \\
$4(1.0$ to $<1.5)$ & 6 & 66.7 & 3 & 2 & 0.100 \\
$5(\geq 1.5)$ & 4 & 64.0 & 3 & 2 & 0.623 \\
\hline
\end{tabular}

$n=$ rounded patient numbers

a The expected morbidity was calculated by multiplying the effective patient number per CRS group by the expected percentage of morbidity previously defined

* $p$ values were calculated using a two-sided exact binomial test. Deceased patients were excluded from morbidity analysis [12]. $p<0.05$ was taken as significant

underestimated with the E-PASS scoring system, particularly for the CRS subgroup analysis. The 44 patients (31.9\%) with a CRS of zero to less than 0.5 had more complications than would have been expected based on the predicted rate (i.e., 28 patients, 20.4\%).

It is evident that the risk of postoperative complications or death is not solely determined by the surgeon's technical skills and his or her manual ability, but also by the patient's physiologic status, the underlying disease necessitating the surgical intervention, and perioperative care [16]. It is the combination of these factors that overtly influences the final outcome. The difficulty lies in trying to determine which patient tolerates which degree of surgical intervention. In the future, this could influence the extent of surgery and the selection of less invasive methods, especially for patients with a high expected CRS ( $>1.0)$, who may be at a greater risk for developing multiple-organ failure [17]. Although a surgeon's instinct is important, obtaining reproducible measures using a preoperative estimate of the expected CRS, based on the calculated PRS and the estimated range of SSS, will allow surgeons to more accurately inform their patients of the potential risks prior to surgery. This estimated preoperative SSS has to be based on personal experience and the average scores obtained from previous similar interventions.

Haga et al. [3] based their initial findings on a very heterogeneous group of patients, of which only a small number $(7.5 \%)$ underwent LR. Further studies validating E-PASS either did not include hepatic surgery [4] or, again, included only a small percentage of patients with LR [12]. We base our findings on a subgroup that underwent only hepatic resection, with only very few selected patients undergoing additional visceral resection. These two patient populations were characterized by a significantly different CRS distribution $(p<0.001)$, with more of our patients belonging to the higher CRS groups.

To minimize postoperative complications, many specialized liver centers currently use computerized tomographic (CT) liver volume measurements to help estimate remnant volume and combine these results with the Child-Pugh score to maximize the assessment of preoperative liver function, which itself has been overtly linked to postoperative liver failure and possible death [18, 19]. Other studies looked at a combination of the ChildPugh score with the indocyanine green retention test and CT volumetry to best predict the short- and long-term outcome after extended LR [20-22]. Although recent findings possibly contradict the long-held belief that postoperative liver failure is the main cause of mortality after major LR, an assessment of preoperative liver function remains the gold standard for patients requiring extensive surgery or for those with varying degrees of cirrhotic liver parenchyma [23]. One might argue that in this setting, the E-PASS model does not take into account the complexity and organ-specific problems unique to hepatic surgery. Because our study population included only a very small percentage $(8.6 \%)$ of patients with cirrhosis, no statistically significant differences were found when comparing the reliability of E-PASS for patients with normal hepatic function to that for patients with reduced hepatic function.

There have been numerous studies to develop audit tools for a variety of surgical specialities, each one trying to accommodate a specific setting [19, 24-30]. The overall advantage of the E-PASS scoring system would seem to be the relative ease with which data are acquired. No special tests are required and intraoperative data collection is limited to a few straightforward measurements. This is favorable to, for example, the POSSUM or P-POSSUM score, which requires 18 different variables compared with the nine variables needed for the E-PASS score, making its use in daily clinical practice tedious and impractical [31, 32].

Other scoring models, such as the acute physiology and chronic health evaluation (APACHE) II score used mainly for patients in intensive care units, take into account only physiologic factors and completely ignore the severity of intraoperative stress. Furthermore, some of these scores, such as the APACHE II score, date back to the mid-1980s 
and the data obtained from this era may not be applicable to the current standard of care [33]. Despite the relative ease with which the E-PASS score can be used, such a score is valuable only if it allows for the reliable acquisition of prognostic data.

In conclusion, the E-PASS scoring system has many advantages such as simple evaluation steps involving easily accessible data and the incorporation of preoperative measurements with equally important intraoperative measurements. The system has already been validated for use in general gastrointestinal surgery and has exhibited a correlation with expected and observed morbidity and mortality. However, the E-PASS scoring system, within the setting of hepatic surgery, cannot be used in its current form and requires further evaluation and validation, with possible adaptations of the original parameters to better fit the postoperative predictions specific to liver surgery.

\section{References}

1. Baugh CW, Bohan JS (2008) Estimating observation unit profitability with options modeling. Acad Emerg Med 15:445-452

2. Conaghan PJ, Figueira E, Griffin MA et al (2002) Randomized clinical trial of the effectiveness of emergency day surgery against standard inpatient treatment. Br J Surg 89:423-427

3. Haga Y, Ikei S, Ogawa M (1999) Estimation of Physiologic Ability and Surgical Stress (E-PASS) as a new prediction scoring system for postoperative morbidity and mortality following elective gastrointestinal surgery. Surg Today 29:219-225

4. Oka Y, Nishijima J, Oku K et al (2005) Usefulness of an estimation of physiologic ability and surgical stress (E-PASS) scoring system to predict the incidence of postoperative complications in gastrointestinal surgery. World J Surg 29:1029-1033

5. Yamashita S, Haga Y, Nemoto E et al (2004) E-PASS (The Estimation of Physiologic Ability and Surgical Stress) scoring system helps the prediction of postoperative morbidity and mortality in thoracic surgery. Eur Surg Res 36:249-255

6. Tang T, Walsh SR, Fanshawe TR et al (2007) Estimation of physiologic ability and surgical stress (E-PASS) as a predictor of immediate outcome after elective abdominal aortic aneurysm surgery. Am J Surg 194:176-182

7. Hirose J, Mizuta H, Ide J et al (2008) Evaluation of estimation of physiologic ability and surgical stress (E-PASS) to predict the postoperative risk for hip fracture in elder patients. Arch Orthop Trauma Surg 128:1447-1452

8. Bone RC, Balk RA, Cerra FB et al (1992) Definitions for sepsis and organ failure and guidelines for the use of innovative therapies in sepsis. The ACCP/SCCM Consensus Conference Committee. American College of Chest Physicians/Society of Critical Care Medicine. Chest 101:1644-1655

9. Boyd O, Grounds RM, Bennett ED (1993) A randomized clinical trial of the effect of deliberate perioperative increase of oxygen delivery on mortality in high-risk surgical patients. JAMA 270:2699-2707

10. Pacelli F, Bossola M, Papa V et al (2001) Enteral vs parenteral nutrition after major abdominal surgery: an even match. Arch Surg 136:933-936

11. Lang M, Niskanen M, Miettinen P et al (2001) Outcome and resource utilization in gastroenterological surgery. Br J Surg 88:1006-1014
12. Haga Y, Wada Y, Takeuchi H et al (2004) Estimation of physiologic ability and surgical stress (E-PASS) for a surgical audit in elective digestive surgery. Surgery 135:586-594

13. Rees M, Tekkis PP, Welsh FK et al (2008) Evaluation of long-term survival after hepatic resection for metastatic colorectal cancer: a multifactorial model of 929 patients. Ann Surg 247:125-135

14. Schiesser M, Chen JW, Maddern GJ et al (2008) Perioperative morbidity affects long-term survival in patients following liver resection for colorectal metastases. J Gastrointest Surg 12:1054-1060

15. Zhou L, Rui JA, Wang SB et al (2007) Outcomes and prognostic factors of cirrhotic patients with hepatocellular carcinoma after radical major hepatectomy. World J Surg 31:1782-1787

16. Copeland GP (2002) The POSSUM system of surgical audit. Arch Surg 137:15-19

17. Haga Y, Ikei S, Wada Y et al (2001) Evaluation of an Estimation of Physiologic Ability and Surgical Stress (E-PASS) scoring system to predict postoperative risk: a multicenter prospective study. Surg Today 31:569-574

18. Wakabayashi H, Ishimura K, Izuishi K et al (2004) Evaluation of liver function for hepatic resection for hepatocellular carcinoma in the liver with damaged parenchyma. J Surg Res 116:248-252

19. Jarnagin WR, Gonen M, Fong Y et al (2002) Improvement in perioperative outcome after hepatic resection: analysis of 1, 803 consecutive cases over the past decade. Ann Surg 236:397-406

20. Bruegger L, Studer P, Schmid SW et al (2008) Indocyanine green plasma disappearance rate during the anhepatic phase of orthotopic liver transplantation. J Gastrointest Surg 12:67-72

21. Inderbitzin D, Muggli B, Ringger A et al (2005) Molecular absorbent recirculating system for the treatment of acute liver failure in surgical patients. J Gastrointest Surg 9:1155-1161

22. Poon RT, Fan ST, Lo CM et al (2002) Extended hepatic resection for hepatocellular carcinoma in patients with cirrhosis: is it justified? Ann Surg 236:602-611

23. Lam CM, Fan ST, Yuen AW et al (2004) Validation of POSSUM scoring systems for audit of major hepatectomy. Br J Surg 91:450-454

24. Kocher HM, Tekkis PP, Gopal P et al (2005) Risk-adjustment in hepatobiliary pancreatic surgery. World J Gastroenterol 11:2450 2455

25. Tekkis PP, Poloniecki JD, Thompson MR et al (2003) Operative mortality in colorectal cancer: prospective national study. BMJ 327:1196-1201

26. Mohil RS, Bhatnagar D, Bahadur L et al (2004) POSSUM and P-POSSUM for risk-adjusted audit of patients undergoing emergency laparotomy. Br J Surg 91:500-503

27. Hadjianastassiou VG, Tekkis PP, Poloniecki JD et al (2004) Surgical mortality score: risk management tool for auditing surgical performance. World J Surg 28:193-200

28. McCulloch P, Ward J, Tekkis PP (2003) Mortality and morbidity in gastro-oesophageal cancer surgery: initial results of ASCOT multicentre prospective cohort study. BMJ 327:1192-1197

29. Tekkis PP, McCulloch P, Steger AC et al (2003) Mortality control charts for comparing performance of surgical units: validation study using hospital mortality data. BMJ 326:786-788

30. Al-Homoud S, Purkayastha S, Aziz O et al (2004) Evaluating operative risk in colorectal cancer surgery: ASA and POSSUMbased predictive models. Surg Oncol 13:83-92

31. Prytherch DR, Whiteley MS, Higgins B et al (1998) POSSUM and Portsmouth POSSUM for predicting mortality. Physiological and Operative Severity Score for the enUmeration of Mortality and morbidity. Br J Surg 85:1217-1220

32. Copeland GP, Jones D, Walters M (1991) POSSUM: a scoring system for surgical audit. Br J Surg 78:355-360

33. Knaus WA, Draper EA, Wagner DP et al (1985) APACHE II: a severity of disease classification system. Crit Care Med 13:818829 